\title{
Norois
}

Environnement, aménagement, société

\section{Aider à construire la légitimité du gestionnaire d'un territoire environnementalement cohérent}

Helping the manager to build his legitimacy over an environmentally consistant territory

\section{Patrick Rio}

\section{(2) OpenEdition}

\section{Journals}

Édition électronique

URL : https://journals.openedition.org/norois/2627

DOI : $10.4000 /$ norois. 2627

ISBN : 978-2-7535-1557-4

ISSN : $1760-8546$

Éditeur

Presses universitaires de Rennes

Édition imprimée

Date de publication : 15 décembre 2008

Pagination : $57-71$

ISBN : 978-2-7535-0805-7

ISSN : 0029-182X

\section{Référence électronique}

Patrick Rio, «Aider à construire la légitimité du gestionnaire d'un territoire environnementalement cohérent », Norois [En ligne], 209 | 2008/4, mis en ligne le 01 mars 2011, consulté le 13 janvier 2022. URL : http://journals.openedition.org/norois/2627 ; DOI : https://doi.org/10.4000/norois.2627

(c) Tous droits réservés 


\title{
AIDER À CONSTRUIRE LA LÉGITIMITÉ DU GESTIONNAIRE D'UN TERRITOIRE ENVIRONNEMENTALEMENT COHÉRENT
}

\author{
PATRICK RIO \\ UMR Lameta INRA \\ (INRA), \\ 2 place Viala - 34060 MonTPELLIER cedex 1 \\ rio@supagro.inra.fr
}

\section{RÉSUMÉ}

La législation environnementale a donné un fort pouvoir d'intervention au gestionnaire chargé localement de la mise en ceuvre des programmes de conservation, de protection ou de mise en valeur en imposant la prise en compte d'un cadre territorial cohérent (zone humide, bassin versant, nappe). Cet état contraste vivement avec les pouvoirs que lui octroient les démarches participatives : des décideurs territorialement impliqués mais dont les préoccupations sont fort éloignés les unes des autres et institutionnellement faiblement articulées. Faire coïncider décision collective et cohérence territoriale suppose que ce gestionnaire maîtrise pleinement l'impact des structures de décision, des règles du jeu, du niveau de représentation des agents concernés. Cette contribution propose d'aider cette démarche en révélant les enjeux locaux à partir de la simulation de différentes structures de négociation incluant la possibilité d'alliance entre parties.

MotS CLÉ : gestion de lagune - négociation - fonction de préférence politique - lagune de Thau-coalition

\footnotetext{
ABSTRACT

Helping the manager to build his legitimacy over an environmentally consistant territory

Imposing an environmentally well defined territorial framework (wetland, catchment, groundwater), the environmental legislation gives a real power to the manager in charge to locally implement the program of conservation, protection or improvement. On the contrary, the participative approaches make him depending on local decision-makers with scattered willingness to reach the environmental objectives. So, for the manager, obtaining a "good decision" implies an accurate view on the local challenges. Some tool can help him to reveal these challenges. We propose such an instrument to simulate how impact on the collective decision (agreement), various negotiation structures including the possibility to deal with alliances among the parties.
}

KEY WORDS : lagoon management - negotiation - political preference function - Thau lagoon - coalition 


\section{Approche intégrée et légitimité du gestionnaire}

La législation relative à l'aménagement du territoire et l'environnement en France favorise les approches participatives (loi Barnier (1995), loi sur l'eau (1992, 2006), loi Chevènement (1999), etc.). Le législateur souhaite impliquer les parties prenantes en vue de stabiliser les décisions dans des domaines où l'information et les préférences des parties sont, localement, fortement engagées. Des structures d'animation : contrat de rivière, schéma de mise en valeur, site Natura 2000, sont mises en place dans ce but. Dans la mesure où la thématique environnementale s'appuie sur une définition scientifiquement fondée du territoire concerné (bassin hydrographique, bassin-versant, cordon littoral, milieu naturel, etc.) donc non discutables, ces structures d'animation et de gestion sont d'abord destinées à faciliter la participation des décideurs concernés. Leur animateur assume un rôle de coordonnateur délicat dans la mesure où la gestion du site nécessite l'obtention d'un accord entre parties prenantes sur lesquelles il n'a pas autorité.

En tant que gestionnaire, la légitimité de cet animateur reposera sur son savoir faire en matière de coordination et notamment sa capacité à comprendre et animer des intérêts locaux multiples, convergents ou contradictoires. Ce travail sera d'autant plus difficile que les détenteurs de l'autorité effective que concède un mandat électif exercent leur prise de décision au sein de dispositifs complexes de délégation. La question du « bon niveau » de négociation est alors posé. Ainsi, pour le bassin-versant de la lagune de Thau auquel nous nous intéresserons, la procédure concertée peut impliquer :

- les 21 maires des communes du bassin-versant,

- leur représentant au sein d'un des 3 Établissements Publics de Coopération Intercommunale (EPCI) qui regroupent 18 communes des 21 communes du bassin ou,

- ces représentants contraints par les objectifs ou les menaces des maires membres de ces EPCI.

La qualité de la gestion participative du territoire concerné exige que les interactions entre parties reflètent exactement les enjeux. Ceci nécessite que soient mis en relief les porteurs des points de vue caractéristiques des intérêts locaux dispersés. Dans ce but, le gestionnaire peut désirer bénéficier d'outils d'analyse lui permettant de simuler l'impact d'un niveau particulier de coordination. Nous avons montré combien l'accord entre décideurs est sensible au choix de la table de négociation, des participants, des règles du jeu (Simon et al., 2007). Simuler diverses structures de négociation apporte donc une information précieuse en vue de stabiliser les accords. Mais surtout, nous montrons ici la possibilité qui nous est donnée de faire saillir de ces simulations, les points de référence les plus signifiants des intérêts locaux.

Dans les paragraphes suivants, nous rappelons les caractéristiques principales de notre outil de simulation d'une démarche négociée, qui se déploie en deux temps : assimilation d'une négociation à un jeu de marchandage qui demande la connaissance des préférences des acteurs puis méthode d'identification de ces dernières. Nous établissons alors notre résultat principal concernant la formation, endogène au jeu de négociation, d'alliances entre parties. Le caractère opérationnel de ces résultats théoriques est ensuite montré en appliquant cet instrument aux questions posées par la gestion du Schéma de Mise en Valeur de la Mer qui s'applique au Bassin Versant de la lagune de Thau. Une attention particulière est portée aux instruments qui facilitent la lecture des résultats obtenus de la mise en œuvre du jeu de marchandage.

L'identification des alliances permet d'évaluer les proximités d'intérêt que les unités élémentaires (les communes du bassin-versant de la lagune de Thau, ici) mobilisent en vue de favoriser leur objectif individuel. Ces proximités sont évaluées par le programme et non données a priori : elles s'expriment en terme d'alliances que les intervenants constituent au cours de la négociation.

La mise en œuvre d'une telle approche a jusqu'ici buté sur deux difficultés :

- passer en revue toutes les coalitions est un problème combinatoire impliquant une grande quantité de calcul $\left(2^{\mathrm{N}}\right.$ opérations, $\mathrm{N}$ étant le nombre de participants à la négociation, soit pour les 21 communes de l'illustration, 2097152 opérations); dans le cas général, cette approche est donc intraitable; 
- si l'évolution des préférences évoluent peu d'une partie du territoire à une partie adjacente, des cycles vont apparaître de la forme 1 propose l'alliance $\{1,2\}, 2$ propose l'alliance $\{2,3\}, 3$ propose $\{3,1\}$ (un participant cherche à former une coalition avec un joueur qui, lui-même, cherche à former une autre coalition, etc.) ; le programme ne peut alors converger.

Dans le cadre négocié proposé, une résolution autorisant la formation des alliances, endogène au problème posé, est possible. Ce résultat est utilisé pour identifier les solidarités naturelles dont le territoire est porteur. Si l'on admet que permettre l'expression de ces solidarités renforce la stabilité de l'accord négocié (un choix de priorité sur les actions à entreprendre), on admettra que cette contribution fournit un complément aux savoir-faire et outils dont le gestionnaire peut disposer.

\section{Approches participatives}

La littérature propose de nombreuses approches participatives qui diffèrent selon le pouvoir de décision qu'elles laissent aux participants. Les plus traditionnelles (l'aménagement du territoire) concevaient la participation comme un apport d'information sur les préférences des parties. Elles ne laissaient pas à ces parties le soin de décider, qui demeure une prérogative centrale. La remise en question théorique et pratique de la conception bienveillante et rationnelle de l'État a conduit à l'étude des conditions sous lesquelles la délégation de la décision est optimale. Ces conditions peu nombreuses impliquent un petit nombre de paramètres :

- le degré d’hétérogénéité des parties (l'homogénéité favorisant les attitudes coopératives),

- le caractère vérifiable de l'information (ce qui favorise les approches argumentatives par opposition à l'échange de points de vue, désirs, positions de principes),

- la valeur de chaque participant dans la procédure de décision lui permettant de penser que son vote peut faire pivoter l'accord d'une proposition à une autre (Persico, 1999).

Réunies, ces conditions invitent à déléguer aux parties le pouvoir de décision, à les doter d'un pouvoir de négociation. L'animateur en charge d'établir la table de négociation détient cependant un pouvoir considérable dans la mesure où l'accord obtenu est sensible à la structure de la négociation : qui est présent, avec quels pouvoirs, pour discuter de quelles variables de décision...

Aider le gestionnaire à concevoir une « bonne table » revient donc simplement à lui donner les moyens de simuler les dynamiques de diverses configurations de cette table de négociation, d'évaluer diverses manières de répartir les pouvoirs d'une assemblée. Dans la mesure où les parties prenantes, (dans notre illustration, les maires, principaux décideurs locaux dans une gestion territoriale) sont rassemblées au sein d'entités plus vastes (dans le cas communal, les EPCI, communautés de communes ou d'agglomération), il existe une certaine latitude pour allouer le pouvoir de décision en fonction du niveau de regroupement retenu, en stipulant éventuellement des conditions de représentation qui s'imposent dès lors qu'un certain niveau d'agrégation est accepté. L'important est là : ces emboîtements de niveaux décisionnels affectent l'élaboration des propositions d'accord. Appuyer l'autorité du gestionnaire reviendra alors à l'aider à évaluer sur simulation l'impact qui résultera du choix des représentants admis à la table des négociations.

Plusieurs descriptions techniques de ces procédures négociées ont été données ailleurs (Rausser et Simon, 1991; Quérou et al., 2007). On peut en donner l'intuition. Considérons une approche de type « ultimatum » : « À prendre ou à laisser. » Il s'agit d'un jeu dans lequel un joueur propose à l'autre un certain partage du gâteau : l'autre répond simplement en acceptant l'offre ou en la refusant. Le répondant est donc confronté à la question de savoir s'il aura « quelque chose » (ce que l'autre joueur lui propose) ou rien, s'il refuse; s'il refuse, ni l'un ni l'autre joueur n'auront quelque chose. Le premier joueur sait donc qu'il n'est pas de son intérêt de tout garder, ce qui conduirait à un résultat d'indifférence pour l'autre joueur qui ne recevrait rien qu'il accepte ou qu'il refuse. Le premier joueur proposera donc quelque chose en partage. En même temps, il sait que l'autre joueur aura intérêt à prendre ce qu'il proposera plutôt que rien. Dans ces circonstances, il 
est rationnel pour le premier joueur de proposer un partage (en pourcentage) de la forme $\{99 \%$, $1 \%$, l'autre joueur n'ayant pas d'intérêt à rejeter cette offre, à perdre le $1 \%$ du gâteau que l'autre joueur lui propose plutôt que rien.

Une procédure de négociation naît lorsque le second joueur peut faire une proposition alternative. Relativement au jeu d'ultimatum, la situation s'inverse, le second joueur, pour les arguments qui valaient pour l'offre du premier, propose le partage symétrique : $\{1 \%, 99 \%\}$. Une solution peut être proposée en tirant au hasard l'une des deux propositions. Supposons que chaque proposition a une chance égale d'être sélectionnée. Alors chaque joueur $i$, en présence de ces propositions, peut espérer gagner $\mathrm{g}_{\mathrm{i}}=1 / 2\{1,99\}+1 / 2\{99,1\}$. Grâce au paradoxe de Allais ( " préférence de la chose sûre »), chacun préférera à cette espérance la proposition (certaine) équivalente $\mathrm{g}_{\mathrm{i}}=\{50,50\}$. Pour que cette dynamique fonctionne, il suffit de supposer, comme dans le jeu de l'ultimatum, qu'en cas de désaccord, une solution « très défavorable » à chacun serait imposée, par une autorité publique par exemple. En pratique, cette clause apparaît souvent comme une option non rendue publique par l'autorité en charge (Thoyer et al., 2004).

L'attribution de probabilités égales aux propositions des deux joueurs est assez naturelle mais peu réaliste. On peut penser en effet que dans une situation réelle les capacités de proposition ne sont pas également réparties mais témoignent des pouvoirs politiques des différentes parties à la table de négociation. Une variation de ce modèle de base consistera à doter les joueurs de pouvoirs de proposition distincts. On peut alors substituer à l'alternance des propositions une procédure simultanée dans laquelle les deux joueurs, au tour pair, annoncent leur préférence (ce serait $\{100,0\}$ et $\{0,100\}$ au premier tour dans ce cas), puis au tour impair, évaluent la possibilité d'annoncer une proposition qui leur soit plus favorable tout en restant admissible par l'autre joueur $(\{50,50\}$ dans ce cas particulier dès le deuxième tour de négociation).

À deux joueurs, ce modèle est d'un intérêt empirique limité. Son intérêt est qu'il est très général, s'applique à un nombre quelconque de joueurs discutant sur un nombre quelconque de variables.

Ces variables étant très diverses, on a besoin de connaître les préférences des joueurs, l’importance qu'ils donnent à ces variables, cette connaissance permettant d'établir leur aspiration, le point idéal que chaque partie à la table de négociation souhaite atteindre. Ce point idéal est la cible dont chaque participant souhaite s'écarter le moins possible, dans le temps même où il accepte de passer des compromis avec les autres parties afin d'éviter de subir le «plus mauvais choix » auquel le condamnerait l'absence d'accord. Aussi devons nous donner au gestionnaire une méthode lui permettant d'identifier les coefficients de préférence que chaque partie applique aux valeurs des variables négociées.

\section{Évaluer les préférences des acteurs}

Les instruments permettant au gestionnaire de construire cette information préalable relèvent souvent des méthodes multicritères. Dans Multi Attribute Value Tree (Hämälainen, 2001), par exemple, les représentants des parties concernées se mettent d'accord sur une liste de critères déclinés en attributs élémentaires : environnementaux, économiques et sociaux, que diverses solutions alternatives sont susceptibles de satisfaire et, par enquête auprès des agents concernés, les différents groupes donnent une priorité aux attributs sélectionnés. Ceci permet d'identifier les éléments de consensus et les éléments conflictuels quant aux systèmes de valeurs portés par la population concernée. Cette démarche est fondée sur une théorie de l'utilité additive (Keeney et Raiffa, 1993) et se prête bien à des situations dans lesquelles les problèmes posés peuvent donner lieu à des projets alternatifs élaborés et contrastés. Les réponses obtenues sont cependant très sensibles aux choix faits sur la décomposition des critères en différents attributs et aux règles d'agrégation retenues pour synthétiser cette information ${ }^{\text {l. }}$.

1. "The spread of weights and the inconsistency between the preference statements depend on the number of attributes that a decision maker considers simultaneously", (Pöyhönen et Hämäläinen, 2001). 
Ceci invite à rechercher une approche plus fruste sans doute mais robuste et bien fondée théoriquement. Celle que nous avons retenue ici repose sur une notion de fonction de préférence politique. L'idée de base est la suivante : contrairement aux hypothèses classiques de la théorie économique qui suppose un régulateur dictatorial omniscient et bienveillant, on admet que les décideurs publics se comportent de manière à optimiser leur chance de réélection et définissent des priorités budgétaires basées sur les intérêts des groupes de soutien les plus favorables (Bullock, 1994). Ce marché politique supposé permet de hiérarchiser différentes orientations politiques comme nous le ferons dans notre illustration.

Taux de croissance communal, développement des équipements de tourisme, équipements publics (école...) et commerciaux (épicerie, boulangerie...), intérêts environnementaux (comme la qualité de l'eau de la lagune ou l'investissement dans les activités d'assainissement), etc. seront évalués en fonction de la répartition de la population communale par Catégories SocioProfessionnelles (CSP). On admet qu'il existe une relation entre les valeurs prises par les différentes dimensions politiques retenues et la répartition de la population. Ceci revient à accepter l'idée que les membres d'une CSP ont des préférences propres indépendantes du lieu dans lequel elles sont localisées, que les priorités politiques retenues sont des signaux qui appellent, maintiennent ou repoussent certaines composantes sociales et, partant, témoignent de la recherche de soutien mise en œuvre par le décideur public. Un intérêt de cette démarche vient du fait qu'elle ne nécessite que des sources d'information faciles à mobiliser (les recensements de population).

Pour obtenir les préférences des composantes sociales relatives aux indicateurs représentatifs des dimensions politiques d'intérêt, on procède par régression linéaire (MCO ou SURE) de ces indicateurs sur leurs valeurs décalées dans le temps et sur la répartition de la population par CSP. Connaissant les préférences des composantes sociales et le poids des CSP localement, on peut évaluer la hiérarchie des préférences de chaque commune sur les dimensions politiques qui font l'objet de la négociation et identifier le point idéal recherché par chaque commune.

\section{Alliances communales autour de la lagune de Thau}

Les diverses facettes de l'instrument d'aide à la décision étant maintenant décrites, nous en illustrerons l'intérêt en l'appliquant aux questions levées par la gestion du Bassin Versant de l'étang de Thau. L'unité hydrographique qui donne sa cohérence au schéma de gestion défini pour la mise en valeur de la lagune est en effet le bassin-versant.

Cette définition implique (tableau 1) de considérer 21 communes dont 18 réparties entre 3 Établissements Publics de Coordination Intercommunale (EPCI), les trois autres communes appartenant à des agglomération hors Bassin, à l'Est de la zone, sous influence directe de l'expansion de Montpellier. Pour faciliter la présentation, nous rassemblerons ces dernières dans un groupe virtuel, «HCB » Hors Communautés du Bassin.

Les enjeux de coordination de ces unités : communes ou EPCI, sont représentés par les diverses options de développement qui se présentent aux communes de la zone. Nous les identifions à quatre orientations politiques : croissance urbaine, croissance touristique, équipement public et commercial, assainissement. Ces différentes dimensions ont été mis en évidence par les travaux réalisés par l'équipe du projet européen Ditty ${ }^{2}$ :

croissance urbaine : elle résulte de la proximité de Montpellier et concerne alors plus spécifiquement les communes situées à l'Est de la zone, déjà fortement intégrées à l'activité de l'agglomération, mais aussi des communes disposant de surfaces constructibles significatives; on notera que Sète génère aussi une demande de logements que les actifs peuvent chercher dans la zone et pas seulement sur la commune;

2. Notamment, les travaux menés sous la direction d'Hélène Rey-Valette qu'elle mit à notre disposition, ce pourquoi nous la remercions ici (Rey-Valette et Valette, 2005). 


\begin{tabular}{|c|c|c|c|c|c|}
\hline EPCI & Commune & $\begin{array}{c}\text { croissance } \\
\text { urbaine }\end{array}$ & $\begin{array}{l}\text { croissance } \\
\text { touristique }\end{array}$ & équipement & $\begin{array}{l}\text { assainis- } \\
\text { sement }\end{array}$ \\
\hline \multirow{3}{*}{$\begin{array}{l}\text { HCB } \\
\text { hors d'un EPCI du } \\
\text { bassin de Thau }\end{array}$} & Aumelas & 0,3820 & 0,0000 & 0,6065 & 0,0114 \\
\hline & Cournonsec & 0,4532 & 0,1374 & 0,2711 & 0,1383 \\
\hline & Fabrègues & 0,4728 & 0,2578 & 0,2693 & 0,0000 \\
\hline \multirow{6}{*}{$\begin{array}{l}\text { CABT } \\
\text { Communauté d'agglo- } \\
\text { mération } \\
\text { du bassin de Thau }\end{array}$} & Balaruc-le-Vieux & 0,3323 & 0,1909 & 0,3573 & 0,1196 \\
\hline & Balaruc-les-Bains & 0,0825 & 0,2803 & 0,6070 & 0,0302 \\
\hline & Frontignan & 0,2378 & 0,2663 & 0,3256 & 0,1703 \\
\hline & Gigean & 0,4099 & 0,2107 & 0,2034 & 0,1760 \\
\hline & Marseillan & 0,0918 & 0,2842 & 0,2954 & 0,3286 \\
\hline & Sète & 0,0886 & 0,6722 & 0,1242 & 0,1151 \\
\hline \multirow{6}{*}{$\begin{array}{l}\text { CCNBT } \\
\text { Communauté de com- } \\
\text { munes } \\
\text { du Nord du bassin de } \\
\text { Thau }\end{array}$} & Bouzigues & 0,3707 & 0,1005 & 0,4032 & 0,1255 \\
\hline & Loupian & 0,1444 & 0,0812 & 0,2738 & 0,5006 \\
\hline & Mèze & 0,2323 & 0,2295 & 0,0911 & 0,4471 \\
\hline & Montbazin & 0,1623 & 0,2707 & 0,3820 & 0,1850 \\
\hline & Poussan & 0,1931 & 0,2016 & 0,4342 & 0,1711 \\
\hline & Villeveyrac & 0,2418 & 0,2842 & 0,3075 & 0,1666 \\
\hline \multirow{6}{*}{$\begin{array}{l}\text { CAHM } \\
\text { Communauté d'agglo- } \\
\text { mération } \\
\text { Hérault Méditerranée }\end{array}$} & Agde & 0,0657 & 0,3013 & 0,4760 & 0,1570 \\
\hline & Castelnau-de-Guers & 0,0307 & 0,3104 & 0,5429 & 0,1160 \\
\hline & Florensac & 0,4097 & 0,5216 & 0,0000 & 0,0687 \\
\hline & Montagnac & 0,2474 & 0,4668 & 0,1692 & 0,1165 \\
\hline & Pinet & 0,2406 & 0,2969 & 0,3332 & 0,1294 \\
\hline & Pomerols & 0,0000 & 0,3686 & 0,4477 & 0,1837 \\
\hline
\end{tabular}

Tableau 1 : Coefficients de préférence des communes du bassin de Thau pour les 4 politiques considérées (sauf indication contraire, les estimations sont de S. Thoyer et P. Rio, 2008)

Preference coefficients of the Than Basin councils for the 4 policies

croissance touristique : principalement sollicitée par les communes du bord de mer (Agde, Marseillan, Sète), elle peut être négativement appréciée face aux intérêts traditionnels relatifs à la protection de la lagune et des activités conchylicoles ou l'amélioration de l'équipement institutionnel et commercial

équipement public et commercial : nous utilisons cette variable pour traduire les tensions qu'une croissance trop soutenue peut provoquer chez les résidents,

assainissement : cette variable mesure indirectement l'attention portée aux activités de pêche et conchylicoles traditionnelles qui sollicitent la protection de la qualité de l'eau de l'étang; on attend une sensibilité particulière des communes limitrophes de l'étang, les retombées touristiques (restauration) et sociales (salariés des mas, artisanat de la pêche) pouvant diffuser cet intérêt sur un espace plus étendu. Rappelons que l'assainissement a constitué la plus grosse dépense inscrite aux budgets des plans successifs de mise en valeur de la mer depuis 1991.

La mesure de ces variables est approchée par les données de l'INSEE et les travaux réalisés dans le cadre du projet Ditty.

Comme on le voit au tableau 1, les préférences communales, au sein de chaque EPCI, sont dispersées. Une analyse rapide de cette dispersion est présentée en annexe. Elle confirme la faible stabilité des EPCI au regard des intérêts locaux des communes concernant les dimensions politiques que nous considérons ici. 
Cette faible stabilité tient à l'hétérogénéité sociale des communes puisque les préférences des composantes sociales (tableau 2) sont supposées identiques sur l'ensemble des 21 communes. Elles ne dépendent pas de la commune de résidence ${ }^{3}$ de celles-ci et les préférences communales résultent de la pondération des préférences de ces composantes sociales en raison de leur poids dans la commune.

\begin{tabular}{|l|c|c|c|c|}
\hline \multicolumn{1}{|c|}{ groupe social } & croissance urbaine & croissance touristique & Equipement & Assainissement \\
\hline Agriculteur & 1,0536 & $-0,5905$ & 0,2823 & $-0,1214$ \\
\hline Artisan & 0,1990 & 0,1515 & 0,9995 & 0,5467 \\
\hline Cadre & 0,6949 & 0,1971 & $-0,8609$ & $-0,5482$ \\
\hline Prof.-Interméd. & $-1,3435$ & $-0,6088$ & 1,4203 & 0,3059 \\
\hline Employé & $-0,0042$ & $-1,2672$ & 0,3826 & $-0,4158$ \\
\hline Ouvrier & $-0,3857$ & $-0,0298$ & 0,8498 & 0,9014 \\
\hline Retraité & $-1,5747$ & 0,4570 & 0,4756 & $-0,1162$ \\
\hline Autre-sans-activité & 1,0663 & 1,3455 & $-1,5125$ & $-0,4442$ \\
\hline Mas (conchyliculteurs) & $-0,2794$ & $-0,1611$ & $-0,1048$ & 0,6580 \\
\hline
\end{tabular}

Tableau 2 : Coefficients de préférence en fonction du groupe social Preference coefficients of the social components

Les données du tableau 2 montrent la variété des attitudes et la qualité de l'information; les ouvriers et les artisans par exemple, dépendants de l'emploi et de l'activité piscicole et conchylicole, manifestent des préférences positives à l'égard de la variable « assainissement » (vue comme une proxy de la place de la conchyliculture), orientées dans le même sens que la variable « mas ». Ce nom désigne un lieu d'exploitation conchylicole. Leur nombre capte la présence des activités conchylicoles.

C'est évidemment l'attrait de l'instrument d'analyse exposé ci-dessus, que de permettre la mise en évidence des solidarités naturelles, en analysant la sensibilité du résultat de la décision collective en fonction de la composition de la table de négociation. Ces solidarités correspondent à l'existence d'intérêts partagés par les communes en fonction de leur localisation ou de leur composition sociale, intérêts que les contraintes imposées par les regroupements institutionnels de type EPCI ne permettent pas de discerner d'emblée. Ces solidarités se repèrent aux alliances que ces communes trouveront si la possibilité leur est donnée d'intervenir dans le processus de négociation hors des cadres institutionnels existant.

Il est facile d'admettre la vraisemblance de tels rassemblements mais, nous l'avons dit, les identifier est une tâche difficile et les résultats présentés ici constituent une première solution dans ce domaine. Comment contourner ces difficultés? Il se trouve que dans notre formulation, les propositions de chaque commune à chaque tour doivent satisfaire la contrainte de participation des autres communes : dans le cas contraire, les autres communes abandonneraient la table de négociation et chacune se verrait contrainte à subir la pire solution. Cette contrainte de participation est évaluée comme l'espérance de gain de chaque autre commune qui dépend des poids politiques de chacune que nous avons assimilés à la probabilité qu'une proposition soit sélectionnée si le jeu venait à s'arrêter. Or, si l'on envisage un processus de vote discret (chaque commune peut remettre son pouvoir à une autre commune mais seulement à une autre commune), cette contrainte peut être autrement définie : une commune A donnera son pouvoir à une autre commune B si cette commune B neutralise par ses choix la contrainte la plus forte qui s'exerce sur A.

3. Les variables exogènes introduites sont : la tendance à long terme de l'urbanisation (qui capte l'intensité de la demande résidentielle du fait de l'urbanisation en place), le revenu imposable, le taux de chômage, les mouvements pendulaires quotidiens rapportés à la population active. 
Partant à chaque tour, il suffit à chacune des $\mathrm{N}$ communes d'évaluer son gain lorsqu'elle donne son pouvoir à chacune des autres communes, ce qui ramène à une complexité d'ordre $\mathrm{N}^{2}$ (420, pour notre exemple) qui ne pose aucun problème de traitement.

En pratique, la prise en compte des solidarités naturelles entre communes revient donc à jouer de deux variables de contrôle que chaque agent invité à la table de négociation possède : une proposition et une allocation des pouvoirs politiques. Ceci peut être illustré maintenant.

\section{Résultats}

L'utilisation « en exploration » du modèle de marchandage multilatéral coalitionnel nous conduit d'abord à choisir un point de vue de référence. En pratique ici, nous nous intéressons aux effets d'agrégation, ce qui nous conduit à opposer deux situations de négociation polaires : celle où les communes n'interviennent que par leur représentant local : l'EPCI, d'une part; celle où ces communes interviennent de manière individuelle, d'autre part. Entre ces deux situations, nous pouvons tenir compte des objectifs des communes au sein de chaque EPCI sur la base d'une négociation locale. Le contenu de ces accords locaux, par EPCI, informe sur l'hétérogénéité des objectifs des parties concernées (tableau 3).

Les communes situées à l'est de la lagune, dans la mouvance de Montpellier (HCB), se focalisent sur la croissance urbaine mais portent une attention soutenue à la politique d'équipement communal; tourisme et assainissement, sont absentes de leurs préoccupations.

Les communes de l'ouest de l'étang (CAHM) recherchent d'abord la croissance touristique et à moindre titre mais fortement l'équipement, plus faiblement la croissance urbaine. L'assainissement est aussi absent de leurs préoccupations.

La communauté d'agglomération du Bassin de Thau (CABT) hiérarchise les objectifs dans l'ordre suivant : équipement, croissance touristique puis croissance urbaine, enfin assainissement dont le poids faible mais positif indique l'intérêt pour certains membres.

Finalement les communes du nord du Bassin de Thau (CCNBT) valorisent l'équipement et, à un niveau élevé, l'assainissement, ce qui témoigne d'une sensibilité et sans doute d'une dépendance plus forte à la qualité des eaux de l'étang. Suivent croissance touristique puis croissance urbaine.

On a donc, entre communauté d'agglomération, des intérêts assez différents, éventuellement spécifiques. On notera comment cette information éclaire sur les difficultés que rencontrera le gestionnaire pour faire émerger un consensus, une vision partagée et stable du développement du territoire concerné : la lagune de Thau.

\begin{tabular}{|l|c|c|c|c|}
\hline accords locaux par EPCI & croissance urbaine & croissance touristique & équipement & assainissement \\
\hline HCB & 1,0000 & 0,0000 & 0,6847 & 0,0000 \\
\hline C ABT & 0,3164 & 0,4352 & 0,5208 & 0,1041 \\
\hline CCN BT & 0,3007 & 0,5806 & 0,8460 & 0,6819 \\
\hline CAHM & 0,3172 & 1,0000 & 0,6216 & 0,0000 \\
\hline
\end{tabular}

Tableau 3 : Accord au sein de chaque EPCI

Agreement by Public Institution for Intercouncils Cooperation

Comparons maintenant les situations pôlaires : la négociation centralisée réunit les 4 EPCI ; la négociation décentralisée réunit les 21 communes (tableau 4). L'effet d'agrégation accroît l'adhésion à chacune des politiques, notamment à la politique d'assainissement qui gagne 0,075 points contre 0,045 à la croissance urbaine, 0,042 à la croissance touristique et 0,039 à la politique d'équipement. L'intérêt de la lagune est mieux défendu dans une structure de négociation centralisée qu'en laissant les communes définir elles-mêmes individuellement leurs objectifs. 


\begin{tabular}{|l|c|c|c|c|}
\hline \multicolumn{1}{|c|}{ structure de négociation } & croissance urbaine & $\begin{array}{c}\text { croissance touris- } \\
\text { tique }\end{array}$ & équipement & assainissement \\
\hline entre EPCI & 0,4795 & 0,5863 & 0,6689 & 0,2567 \\
\hline EPCI sous PI-controle & 0,4868 & 0,5192 & 0,6906 & 0,1578 \\
\hline EPCI sous PM-controle & 0,5969 & 0,5080 & 1,0000 & 0,1899 \\
\hline communes individuellement & 0,4336 & 0,5448 & 0,6300 & 0,1817 \\
\hline $\begin{array}{l}\text { communes avec possibilité de } \\
\text { coalition }\end{array}$ & 0,4342 & 0,5699 & 0,5986 & 0,2334 \\
\hline
\end{tabular}

Tableau 4 : Accord en fonction de la structure de la négociation Agreement depending of several negotiation structures

L'effet de l'agrégation sera mieux compris en étudiant l'impact des contrôles que les communes peuvent exercer sur les négociations menées au niveau des EPCI (tableau 4). On considère deux cas :

- dans le premier cas, chaque EPCI prend pour point idéal (PI) l'accord obtenu entre les communes qui en sont membres (tableau 3) - ce cas sera dit «PI-contraint »;

- dans le second cas, l'utilité de l'accord entre communes-membres de l'EPCI (tableau 4) sera une borne explicite pour chaque négociateur et constituera un point de retrait : toute proposition qui aboutirait à une utilité moindre, sera rejetée par l'EPCI dans la négociation avec les autres EPCI. Ce cas sera dit «PM-contraint» car elle correspond à une contrainte de participation définie par un point de menace (PM) lorsque ce point de menace est plus contraignant que l'espérance d'utilité. Cette menace a priori laisse plus de place à un accord terminal, car la contrainte ne s'exerce pas sur l'écart pris relativement à la proposition traduisant le compromis local mais par rapport à l'utilité de cette proposition commune, ce qui permet des compensations entre les différentes dimensions de la politique.

Dans les deux cas, mais de manière plus marquée dans le second cas de figure (PM-contraint), l'accord favorise la croissance urbaine et la politique d'équipement, au détriment de la croissance touristique et surtout de l'assainissement. Autrement dit, plus les communes s'affirment individuellement ou au travers de leur représentant (EPCI) qu'elles contrôlent étroitement, moins une politique de gestion environnementale sera acceptée.

Il est alors particulièrement intéressant de noter comment, en donnant aux communes la possibilité de se coaliser, les communes accorderont une attention accrue à la politique d'asssainissement. Il faut y voir l'effet propre à la coalition des communes riveraines (Marseillan, Mèze, Loupian). Bien que ce résultat évoque l'accord atteignable par les EPCI, on ne peut confondre ce résultat avec celui d'une négociation centralisée. Au contraire, on met en évidence un respect plus grand des options communales quant au contrôle de la croissance urbaine et de la politique d'équipement. La différence vient donc surtout de la structure spatiale implicite que révèlent les alliances entre communes qui se distingue aussi bien des effets de contiguïté qu'impose le cadre spatial fixé par la délimitation des EPCI que du traitement indifférencié des communes dans l’approche décentralisée.

\section{Outils de lecture des résultats}

Insistons maintenant sur les outils qui faciliteront la lecture des résultats fournis par le modèle de marchandage.

Le premier tableau de travail (tableau 5) associe à chaque commune (numérotée dans l'ordre alphabétique) la liste des bénéficiaires de l'allocation de leurs droits de vote à chaque tour de négociation. Par exemple, Agde cède son pouvoir à la commune 9 (Florensac) au dernier tour de négociation, commune dont les préférences vont à la croissance touristique (voir annexe). 


\begin{tabular}{|c|c|c|c|c|c|c|}
\hline & \multirow{2}{*}{$\begin{array}{l}\text { Numéro de } \\
\text { commune }\end{array}$} & \multicolumn{5}{|c|}{ tour de négociation } \\
\hline & & 1 & 2 & 3 & 4 & 5 \\
\hline Agde & 1 & 1 & 1 & 1 & 1 & 9 \\
\hline Aumelas & 2 & 2 & 2 & 2 & 2 & 2 \\
\hline Balaruc le Vieux & 3 & 3 & 5 & 5 & 5 & 1 \\
\hline Balaruc les Bains & 4 & 4 & 4 & 4 & 4 & 1 \\
\hline Bouzigues & 5 & 5 & 2 & 2 & 3 & 2 \\
\hline Castelnau de Guers & 6 & 6 & 1 & 1 & 1 & 14 \\
\hline Cournonsec & 7 & 7 & 7 & 2 & 3 & 2 \\
\hline Fabrègues & 8 & 8 & 8 & 8 & 2 & 2 \\
\hline Florensac & 9 & 9 & 9 & 9 & 8 & 7 \\
\hline Frontignan & 10 & 10 & 10 & 10 & 4 & 4 \\
\hline Gigean & 11 & 11 & 8 & 8 & 2 & 2 \\
\hline Loupian & 12 & 12 & 12 & 12 & 12 & 12 \\
\hline Marseillan & 13 & 13 & 13 & 13 & 12 & 12 \\
\hline Mèze & 14 & 14 & 14 & 14 & 12 & 7 \\
\hline Montagnac & 15 & 15 & 20 & 9 & 9 & 7 \\
\hline Montbazin & 16 & 16 & 13 & 1 & 1 & 9 \\
\hline Pinet & 17 & 17 & 17 & 1 & 1 & 14 \\
\hline Pomerols & 18 & 18 & 1 & 1 & 1 & 9 \\
\hline Poussan & 19 & 19 & 4 & 4 & 4 & 14 \\
\hline Sète & 20 & 20 & 20 & 9 & 8 & 7 \\
\hline Villeveyrac & 21 & 21 & 17 & 17 & 1 & 12 \\
\hline
\end{tabular}

Tableau 5 : Ré-allocation des droits de votes (estimations de l'auteur) Voting rights allocation

Cette information est synthétisée graphiquement par la construction d'un graphe orienté (fig. 1). Chaque commune constitue un nœud du graphe et l'existence d'une relation entre deux communes est une arête. Cette structure est ici aisément intelligible parce que le graphe résultant est planaire (sans intersection). Ces relations sont dirigées, répartissant les communes entre « émetteurs » (qui donnent leur pouvoir) et « récepteurs » (dont la proposition se substitue à celle de l'émetteur).

- Les «émetteurs purs» ne représentent aucune autre commune qu'elles-mêmes. Dans ce groupe, on range 8 communes : 6 (Castelnau de Guers), 10 (Frontignan), 11 (Gigean), 15 (Montagnac), 16 (Montbazin), 18 (Pomerols), 19 (Poussan) et 21 (Villeveyrac); globalement ces communes sont les moins engagées dans la problématique lagunaire, les plus agricoles ou déjà impliquées dans l'expansion Montpelliéraine; on notera que plusieurs d'entre elles délèguent leur pouvoir entre des orientations très différentes selon le tour de jeu : lagunaire et touristique / urbaine et touristique...

- Les « récepteurs purs » reçoivent les intentions de vote d'autres communes et, de ce fait, constituent des orientations archétypiques. Elles sont deux : 2 (Aumelas) et 12 (Loupian), et synthétisent les comportements polaires centrés sur la qualité de la lagune (Loupian : préférence la plus forte pour l'assainissement) ou les dynamiques internes (équipement) et urbaines (Aumelas).

- Entre ces deux classes, quelques communes constituent des références comme 1 (Agde), 7 (Cournonsec), 9 (Florensac) et 14 (Mèze), mais optent elles-mêmes en faveur d'autres représentants qui, in fine, les rattachent à l'une des deux orientations archétypiques. 
Figure 1 : Représentation sous forme de graphe presentation

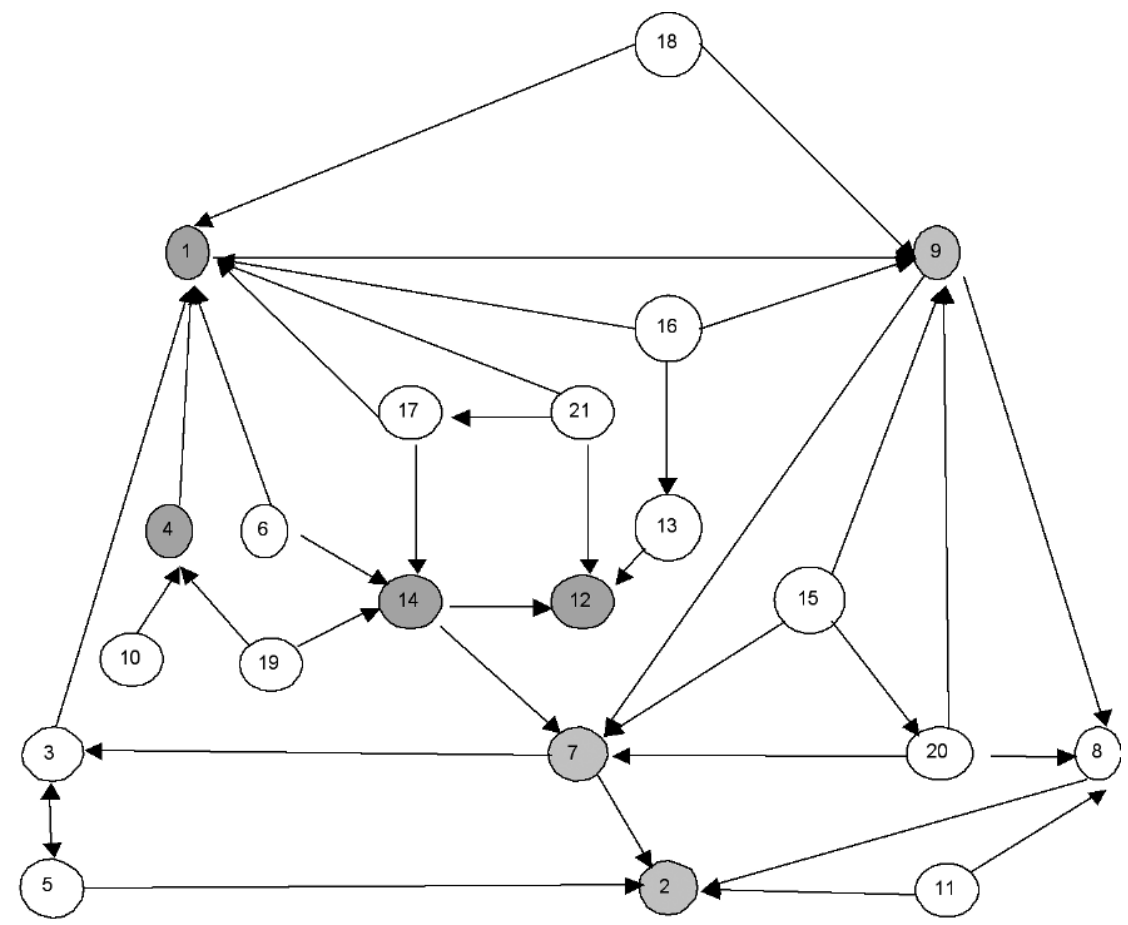

Cette lecture est enrichie par une projection cartographique de cette information (fig. 2).

Cette représentation met en évidence la fréquence des relations distantes, des soutiens donnés ou reçus de l'ouest à l'est de la zone : il est clair que des dynamiques communes existent qui ne s'expriment pas dans le découpage en communautés d'agglomération. Dans le même temps, cette représentation spatiale des liens témoigne d'une réalité spatiale et sociale de la zone concernée.

Les deux orientations polaires apparaissent comme de vrais clivages spatiaux, Loupian structurant l'espace intérieur, central de la zone, Aumelas captant les relations externes, est-ouest. Entre ces deux ensembles, une ligne de fracture représentée par les communes "émetteurs purs ", comme un croissant traversant cet espace du nord-ouest (Castelnau de Guers) au sud-est (Frontignan) en passant par Montagnac, Villeveyrac, Poussan, Montbazin et Gigean.

Une zone ambiguë à fort pouvoir référentiel pour les communes de la zone est constituée à l'ouest de ce territoire par les deux communes adjacentes d'Agde et de Florensac. En dépit de l'existence de liaisons multiples avec et entre ces deux communes selon le tour de négociation, on observe que l'attraction de chacune est opposée : vers les questions lagunaires pour Agde (lien avec «Loupian », via Mèze); vers la dynamique urbaine pour Florensac («Aumelas », via diverses alliances avec Cournonsec et Fabrègues). Cette ambiguïté peut refléter l'existence d'une problématique commune aux participants, qui ne se distinguent que marginalement sur les thématiques considérées.

Cette approche conduit à s'interroger sur la dynamique de Sète, portée par les aspects urbains plus que par les aspects lagunaires, par opposition notamment avec Marseillan qui, avec Mèze et Loupian, forme le noyau dur d'une ligne de mise en valeur de l'étang.

Finalement, un noyau d'intérêt se perçoit autour de Bouzigues, Balaruc-le-Vieux, Balaruc-les-Bains. L'approche lagunaire y est peu présente. La croissance urbaine mais aussi l'équipement communal sont les aspects principaux de relations dispersées : Cournonsec, Aumelas mais aussi Agde. 


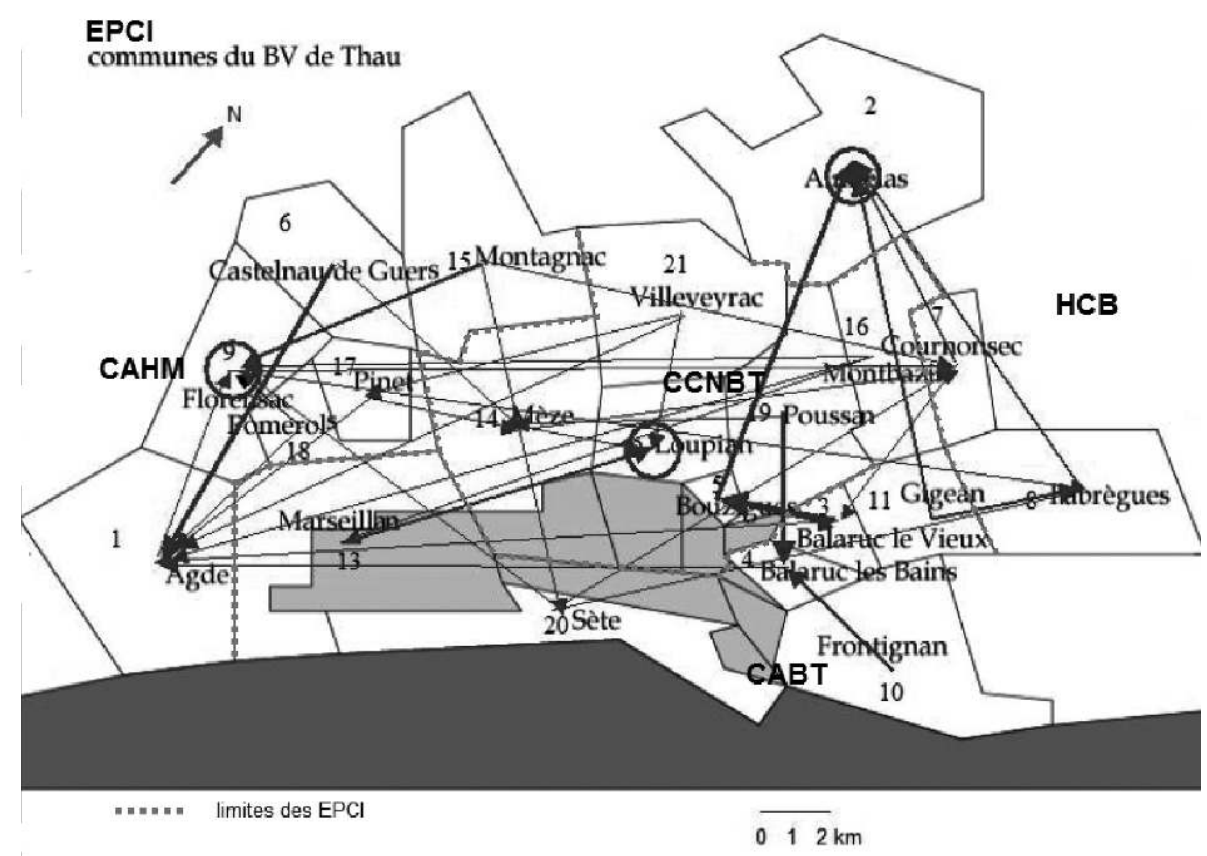

Figure 2 : Projection cartographique des liens entre communes (les numéros renvoient au tableau 5) Mapping of the councils links (numbers refer to table 5)

Passer du graphe à une représentation matricielle nous ouvre l'accès aux nombreuses méthodes d'analyse des données. Nous l'illustrons par une analyse de type Bertin consistant à redistribuer les lignes et colonnes d'un tableau pour en faire ressortir les caractéristiques dominantes (Bertin, 1980) qui souligne certains résultats de l'analyse des liens menée ci-dessus.

Cette représentation met en relief l'existence d'« émetteurs purs » (les 8 dernières colonnes) et de «récepteurs purs» (les deux dernières lignes) (tableau 6). L'existence de sous-diagonales fait penser à un continuum des liens traduisant un fonds de problèmes communs. On observe aussi l'intensité de la référence Agde (1) et, à moindre titre, de Florensac (9) qui renvoie à des liens plus dispersés.

De nombreux autres outils d'analyse de données basés sur une diagonalisation de la matrice des liens sont mobilisables: AFC, ACP-VI... Pour variables instrumentales, typiquement, on mobilisera une matrice d'adjacence et un vecteur d'allocation des communes aux différents EPCI, pour extraire la composante « contiguïté spatiale » de l'information obtenue sur les liens. Mais ces approches, classiques, méritent moins de développement ici.

\section{Conclusion}

La législation environnementale valorise les approches intégrées. Ce terme renvoie d'abord à la définition rationnelle du système physique environnementalement concerné et de ses limites. L'unité de territoire est cependant moins claire quand on tente de l'exprimer du point de vue de la prise de décision : la hiérarchie des actions justifiée sur le plan environnemental n'a pas de raison a priori de coïncider avec celle que les décideurs politiques ou économiques sont désireux de construire localement. Cette hiérarchie des actions n'a pas non plus vocation a priori à être partagée par ces communes, individuellement ou groupées au sein des Communautés d'agglomération ou autres EPCI. 


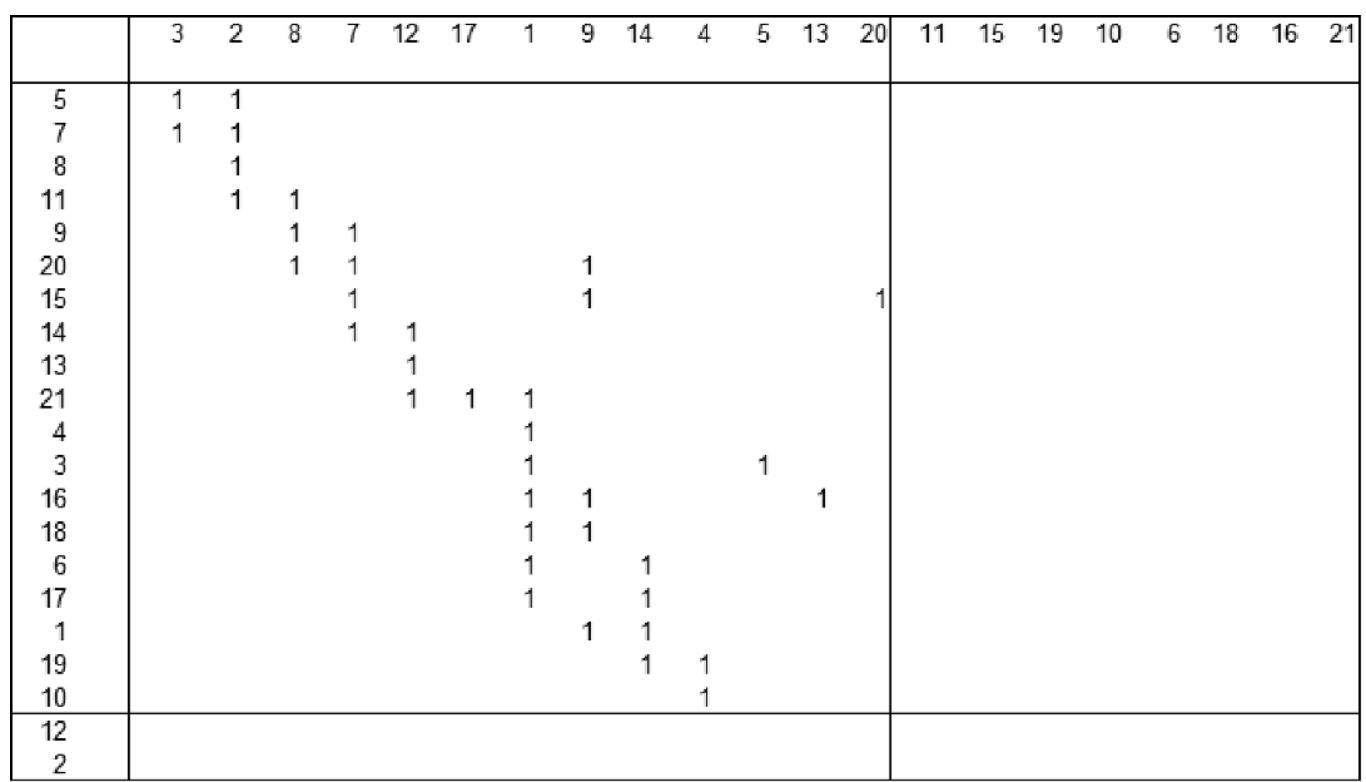

Tableau 6 : Représentation matricielle du graphe des relations après réorganisation des lignes et des colonnes [approche Bertin] (la liaison d'une commune avec elle-même est tenue pour nulle; une liaison nulle est exprimée par une cellule vide)

Matrix presentation of the previous graph with row-and column-ordering [Bertin's approach] (a council has a 0-link with itself; a O-link is expressed by a void cell)

Cette situation va conduire le gestionnaire à mettre en œuvre des procédures de coordination et notamment parmi les procédures participatives disponibles, des approches négociées dans le but d'harmoniser les objectifs environnementaux «scientifiquement » fondés et des objectifs locaux très dispersés. C'est le prix qu'il devra payer pour instruire face aux responsables locaux sa légitimité pour le territoire concerné. Compte tenu de l'importance de l'enjeu, l'intérêt de disposer d’instruments simulant de telles procédures négociées est évident.

Après avoir décrit les méthodes permettant de refléter les intérêts des parties concernées, la nature des informations collectées dans ce cadre a été rappelée. Nous avons alors souligné la pertinence d'une information permettant d'appréhender les solidarités naturelles des parties, et avons fourni une solution pour rendre ce problème traitable. Elle repose sur la révélation que nous permettons, des alliances que génère la négociation, qui expriment ces solidarités entre les parties.

Nous appliquons alors cette approche aux 21 communes concernées par la mise en valeur de la lagune de Thau et aux différents niveaux de regroupement permis par la donnée de 3 EPCI (+1 artificiel). L'algorithme proposé permet ce traitement qui ne pourrait être envisagé pour un si grand nombre de parties dans une approche coalitionnelle standard.

Les résultats obtenus soulignent la sensibilité des accords à la structure de négociation choisie et notamment l'intérêt des structures qui laissent les communes former des alliances, une configuration favorable en matière environnementale. Nous les mobilisons aussi pour mettre en évidence l'existence d'outils d'analyse de ces alliances qui facilitent grandement la compréhension des convergences et conflits d'intérêt au sein d'un territoire. Ceci conduit à des programmes de traitement de données faciles à développer et il y aurait un grand intérêt à observer le résultat de leur mise en ouvre dans d'autres territoires. 


\section{Annexe}

\section{CLASSIFICATION DES COMMUNES EN FONCTION DE LEURS PRÉFÉRENCES ESTIMÉES}

Sur la base des préférences communales estimées, il est possible d'évaluer la cohérence des regroupements communaux au regard des dimensions des politiques communales que nous avons sélectionnées : croissance urbaine, croissance touristique, équipement public et commercial et assainissement. L'outil de classification utilisé pour ce regroupement est construit à partir des méthodes de classification floues proposées par N. R. Pal et J. C. Bezdek (1995). À la différence des classifications usuelles, qui affectent chaque observation à une classe, on calcule ici des possibilités d'appartenance, c'est-à-dire des proximités au noyau de chaque classe. Le classement initial repose sur le profil des préférences moyennes des EPCI.

Le reclassement permet de souligner la faible stabilité des regroupements institutionnels au regard de ces préférences, sauf pour les communes hors Communautés du Bassin. Ces regroupements renforcent plutôt les traits saillants des profils initiaux,

- en faisant apparaître une exigence forte en faveur de l'assainissement chez les communes limitrophes de l'étang : Marseillan, Loupian, Mèze;

- en accusant la demande d'équipement institutionnel et commercial (Balaruc-les-Bains, Sète, Agde, Castelnau-de-Guers et Pomerols).

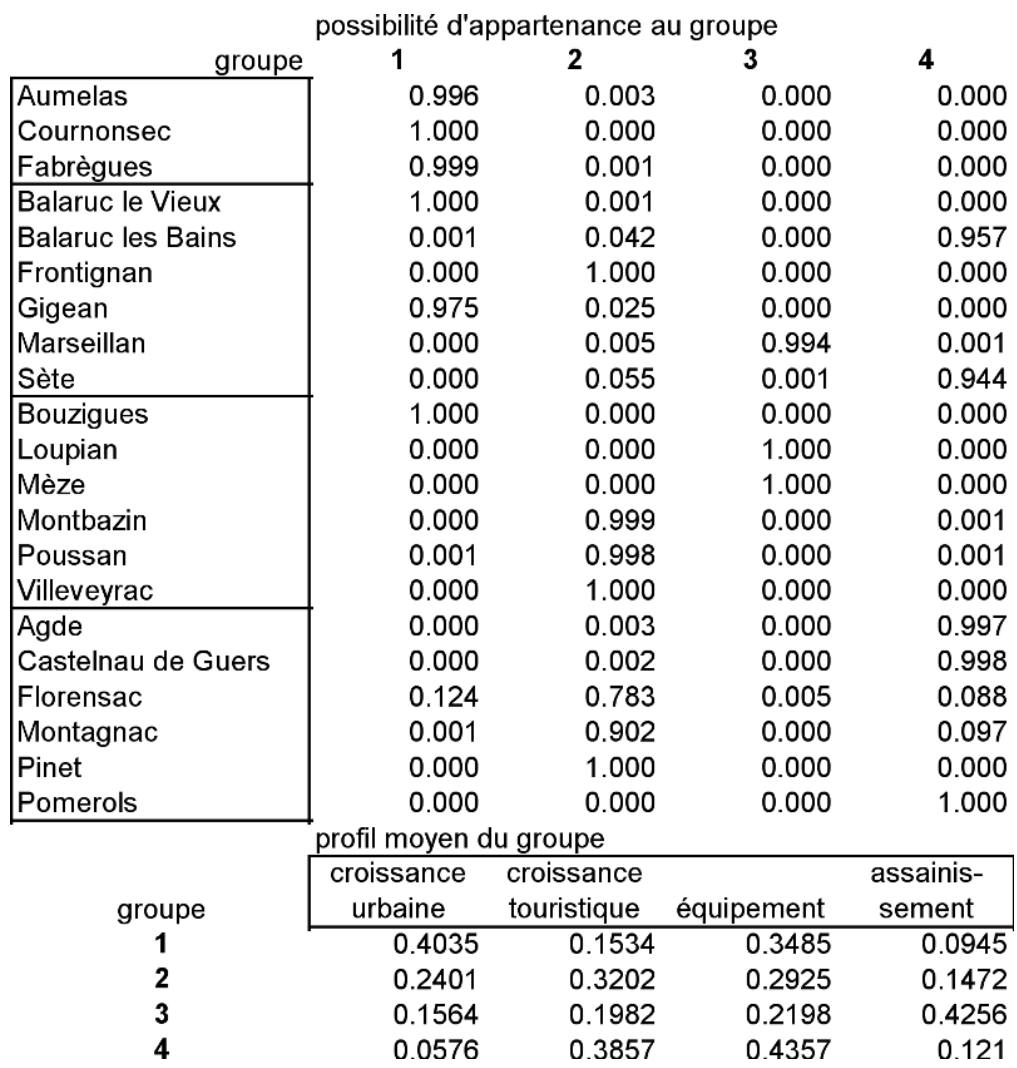

Classification floue des communes selon leurs préférences : possibilité d'appartenance et profil de préférence moyen des classes obtenues Fuzzy classification of the councils depending on their preferences : membership possibility and mean class profiles of preferences 


\section{Bibliographie}

Bertin (J.), 1980. - « Traitements graphiques et mathématiques. Différence fondamentale et complémentarité ». Mathématiques et sciences humaines, tome 72, p. 60-71.

Bullock (D. S.), 1994. - « Insearch of Rational Government : What Political Preference Function Studies Measure and Assume », Amer. J. Agr. Econ., n 76, p. 347-361.

Hämäläinen (R. P.), Kettunen (E.), Marttunen (M.), Ehtamo (H.), 2001 - - « Evaluating a Framework for Multi-Stakeholder Decision Support in Water Resources Management », Group Decision and Negotiation, vol. 10, p. 331-353.

KeEney (R. L.), Raiffa (H.), 1993. - Decisions with multiple objectives-preferences and value tradeoffs, Cambridge/New York, Cambridge University Press, 569 p.

Pal (N. R.), Bezdek (J. C.), 1995. - « On Cluster Validity for the Fuzzy c-Means Model », /TFS/, 3(3), p. $370-379$.

Persico (N.), 1999. - « Consensus and the Accuracy of Signals: Optimal Committee Design with Endogenous Information », Penn CARESS Working Papers 45c846ad903decd7bf77blc66, UCLA Dep. of Economics, revised, [http://www.ssc.upenn.edu/econ/CARESS/CARESSpdf/99-08.pdf].

PÖYHÖNEn (M.), HÄMÄLÄINEN (R. P.), 2001. - « On the Convergence of Multiattribute Weighting Methods », European Journal of Operational Research, vol. 129, n³ January 2001, p. 106-122.

Quérou (N.), Rio (P.), Tidball (M.), 2007. - « Multi-Party Negotiation When Agents Have Subjective Estimates of Bargaining Powers », Group Decision and Negotiation, vol. 16, n 5, p. 417-436.

Rausser (G.), Simon (L.), 1991. - «A noncooperative model of collective decision making: A multilateral bargaining approach », Working Paper n. 618, Department of Agricultural and Ressource Economics, University of California, Berkeley, $50 \mathrm{p}$.

Rey-Valette (H.), Valette (F.), 2005. - « Contribution à la définition d'une politique de gestion intégrée de la zone de Thau, étude prospective des scénarios », WP7, Études d'appui à la définition des scénarios, Rapport DITTY, 112 p.

Simon (L.), Goodhue (R.), Rausser (G.), Thoyer (S.), Morardet (S.), Rio (P.), 2007. - « Structure and power in multilateral negotiations: an application to French water policy », Giannini Foundation Monograph Series (47), 46 p.

Thoyer (S.), Morardet (S.), Rio (P.), Simon (L.), Goodhue (R.), Rausser (G.), 2001. - «A bargaining model to simulate negotiations between water users », Journal of Artificial Societies and Social Simulation, vol. 4, n 2 [http://www.soc.surrey.ac.uk/JASSS/4/2/6.html].

Thoyer (S.), Morardet (S.), Rio (P.), Goodhue (R.), 2004 - - "Comparaison des procédures de décentralisation et de négociation de la gestion de l'eau en France et en Californie », Natures Sciences Sociétés, $n^{\circ} 12$, p. 7-17.

Thoyer (S.), Rio (P.), 2008. - «Simulation des négociations et intercommunalité sur l'Étang de Thau », Document de travail LAMETA, $24 \mathrm{p}$.

Cet article a été reçu le 12 avril 2008 et définitivement accepté le 5 novembre 2008. 